\title{
EXPRESSION OF MIRNAS SUGGESTS A POTENTIAL ROLE IN BREAST CANCER
}

Tamyres MIngorance Carvalho', Tayana Schultz Jukoski', Guillermo Ortiz Brasil'1, Flavia Kuroda², Enilze M. S. F. Ribeiro

${ }^{1}$ Universidade Federal do Paraná - Curitiba (PR), Brazil.

${ }^{2}$ Hospital Nossa Ssenhora das Graças - Curitiba (PR), Brazil.

Introduction: MicroRNAs (miRNAs) are regulators of gene expression in biological processes, mainly repressing translation or degrading messenger RNA (mRNA) from their target genes. Their deregulation is associated with a wide variety of diseases, including cancer. Breast cancer $(\mathrm{BC})$ is the most common cancer among women worldwide. Understanding the mechanisms involved in this pathology is essential for the discovery of new diagnostic and prognostic markers. Objectives: Investigate the differential expression of selected miRNAs in luminal A (LA) and triple-negative breast cancer (TNBC). Methods: We evaluated the expression of miR-320a, miR-4433b-5p, miR-142-5p, and miR-150-5p in 31 BC samples (19 LA and 12 TNBC) and 29 adjacent non-tumor breast cancer (NT). The miRNAs were selected after in silico study. RT-qPCR was the method of choice, using RNU48 as endogenous control, and the BT-474 cell line was used as a calibrator between plates. The $2-\Delta \Delta \mathrm{Ct}$ method was used to estimate miRNA expression level. Individual receiver operating characteristic (ROC) curves were calculated based on RQ values to investigate the diagnostic value of miRNAs. Results: miR-142-5p, miR-150-5p, miR-320a, and miR-4433b-5p were downregulated in BC compared to NT samples. All studied miRNAs were able to discriminate between BC and NT samples with sensitivity and sensibility (AUC - Area under curve $>0.7$ and $\mathrm{p}<0.05$ ). A panel including all miRNAs improved the AUC to identify TNBC patients compared to NT (AUC=0.9240, sensitivity $94.44 \%$, specificity 100\%). We found no difference comparing miRNAs between LA and TNBC BC subtypes. There was no association between expression levels and prognostic parameters (age, histological grade, size of the tumor, axillary lymph node status). Conclusions: Our data suggest that downregulation of miR-142-5p, miR-150-5p, miR-320a, and miR-4433b-5p can be involved in BC for not repressing the expression of target genes. Functional studies will contribute to elucidate their involvement in $\mathrm{BC}$. 